\title{
Primary cutaneous plasmacytosis: a case report
}

Adelina $\operatorname{Costin}^{1 凶}$, João Alves ${ }^{1}$, Diogo Cerejeira ${ }^{1}$

\begin{abstract}
Primary cutaneous and systemic plasmacytosis is a rare disorder characterized by infiltration of the skin by polyclonal plasma cells of unknown etiology, frequently accompanied by polyclonal hypergammaglobulinemia and diffuse superficial lymphadenopathy. It primarily arises in patients of Japanese descent, and it is exceedingly rare in Caucasians. We describe a 36-year-old Portuguese male who presented with disseminated reddish-brown plaques over the trunk, neck, and upper limbs with normal gammaglobulinemia consistent with a diagnosis of primary cutaneous plasmacytosis.
\end{abstract}

Keywords: cutaneous plasmacytosis, plasma cell dyscrasia

Received: 12 April 2019| Returned for modification: 31 July 2019 | Accepted: 19 August 2019

\section{Introduction}

Primary cutaneous plasmacytosis is a rare reactive plasmacytic disorder characterized by infiltration of the skin by mature polyclonal plasma cells. Initially thought to represent an exclusively cutaneous process, it was renamed primary cutaneous and systemic plasmacytosis due to the observation of frequent extracutaneous involvement (1). It primarily arises in patients of Japanese descent, with fewer than a dozen reports in Caucasians (2). The pathogenesis is unclear and is thought to be related to an unspecific reactive inflammatory response.

\section{Case report}

An otherwise healthy 36-year-old Caucasian male presented to our outpatient clinic with a history of 10 years' duration of progressive development of slightly pruritic hyperpigmented lesions that began on his upper trunk and gradually spread to the entire trunk, neck, and upper limbs. He denied fever, malaise, night sweats, or other constitutional symptoms and he did not have a relevant family history of hematologic disorders. On physical examination he presented with multiple reddish-brown ovoid thin plaques, up to $2 \mathrm{~cm}$ in size on his back, chest, neck, and upper limbs (Figs. 1-3). Neither lymphadenopathy nor hepatosplenomegaly was appreciated clinically.

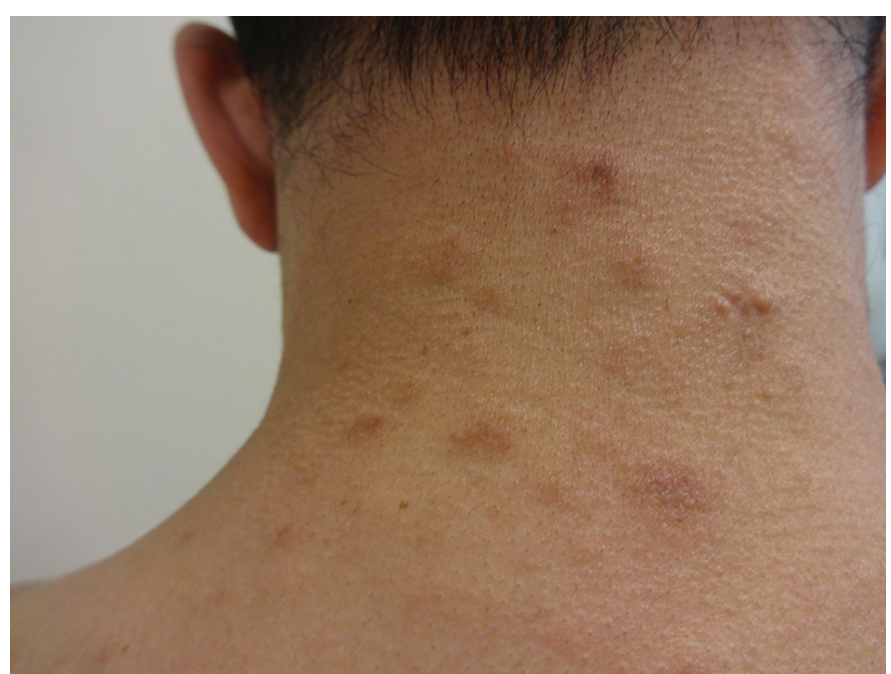

Figure 1 | Erythematous to brownish plaques on the posterior neck.

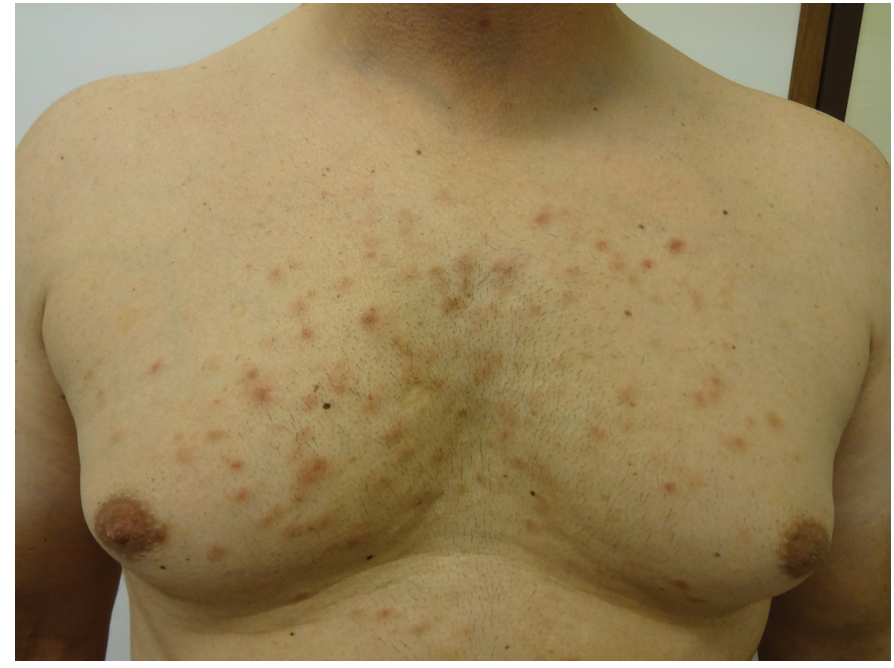

Figure 2 | Erythematous to brownish macules and plaques on the anterior chest.

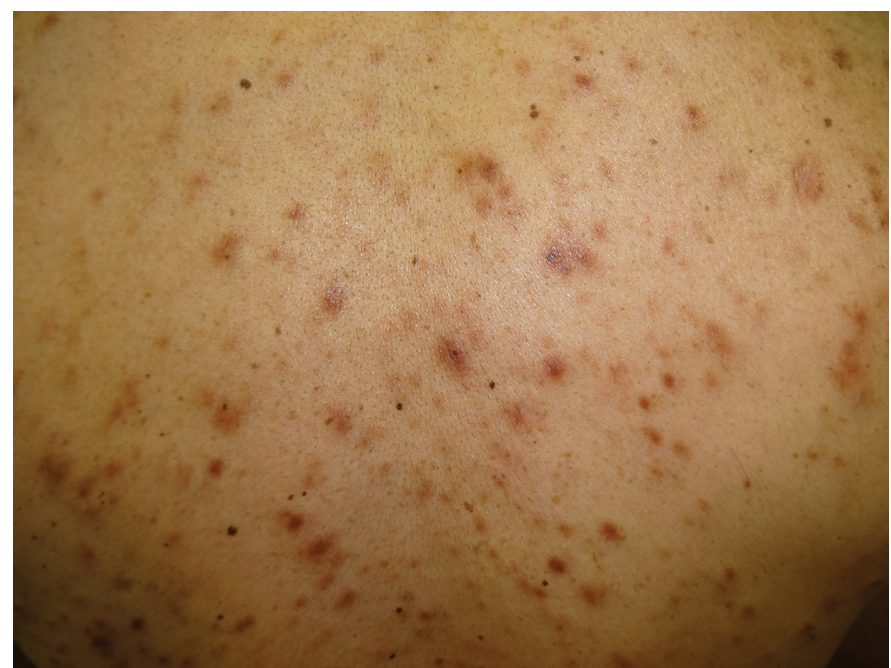

Figure 3 | Detail of erythematous to brownish plaques on the trunk.

Serum and urine electrophoresis were within normal range, with a normal IgG value ( $637 \mathrm{mg} / \mathrm{dl})$. Complete blood count, metabolic panel, antinuclear antibodies, and erythrocyte sedimentation rate revealed no alterations, with normal beta-2 microglobulin and interleukin-6 (IL-6) levels (<2.0 pg/ml). Serum and urine immunofixation studies found no monoclonal immunoglobulins. The serological examination for syphilis, hepatotropic viruses, HIV, and Borrelia burgdorferi was negative. 
Chest computed tomography and abdominal ultrasound were performed, with no changes disclosed. Bone marrow aspiration revealed normocellularity ( $<5 \%$ of plasma cells).

A punch biopsy was obtained from a cutaneous lesion. The histological examination revealed a dense perivascular inflammatory cell infiltrate in superficial and deep dermis, with predominance of mature plasma cells intermingled with some lymphocytes, histiocytes, and eosinophils (Fig. 4). The plasma cells displayed no atypia. Immunohistochemical study showed coexistence of both kappa and lambda-chain positive cells, thus confirming the polyclonal nature of plasma cells.

Neither topical corticosteroids nor topical tacrolimus was effective. The patient declined a psoralen and ultraviolet A (PUVA) therapy trial, remaining in clinical follow-up since then.

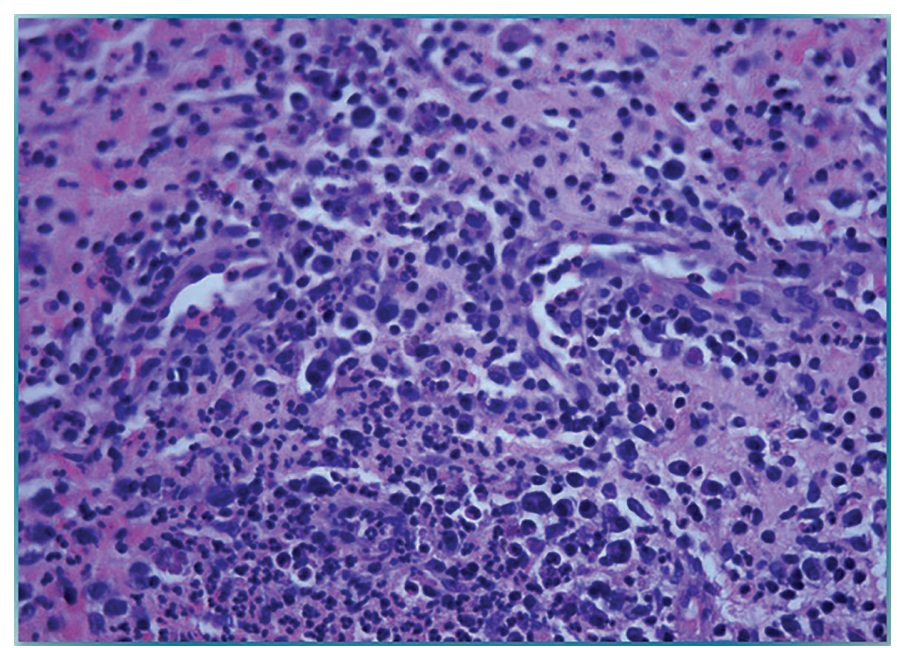

Figure 4 | Dense perivascular inflammatory infiltrate in the superficial and deep dermis with mature plasma cells without atypia (H\&E 400x).

\section{Discussion}

The clinical presentation of primary cutaneous plasmacytosis is characteristic, with gradual development of multiple reddishbrown plaques and nodules predominantly on the trunk (3).

It mainly affects middle-aged patients of Asian descent, without a clear sex predilection (4). Less frequently it can present as a solitary lesion, not usually associated with extracutaneous manifestations and occurring predominantly in children (5).

Idiopathic infiltration of mature polyclonal plasma cells has been reported not only in the skin but also in other organs, such as the lungs, lymph nodes, liver, and spleen, in which case it is referred to as primary cutaneous and systemic plasmacytosis (6). The most frequent extracutaneous manifestations are superficial lymphadenopathy (up to 58\%) and hypergammaglobulinemia (up to 93\%) (1).

There are several secondary causes of mature plasmacytic infiltrates that should be excluded, such as collagen vascular disease and chronic infections. A main differential diagnosis is generalized cutaneous B cell pseudolymphoma due to their striking histological similarities. Extracutaneous involvement such as lymphadenopathies, hypergammaglobulinemia, and no previous history of culprit drug intake are important clues for establishing the diagnosis.

Whereas the exclusively cutaneous forms follow a benign and chronic course without spontaneous remission, in some cases with systemic involvement it can follow an aggressive course, with cases of lymphoid interstitial pneumonia and mesangial proliferative glomerulonephritis and renal insufficiency having been reported (4). The potential for malignant transformation of cutaneous plasmacytosis is not known. The few reports of lymphoma do not establish a correlation, and the relationship between lymphoma and plasmacytosis is not established (7).

Due to its rarity and most of the literature consisting of case reports and case series, the etiology and therapeutic approaches remain poorly established. Although the exact pathogenesis is unknown, increased serum levels of IL- 6 have been reported in these patients, similarly to multicentric Castleman disease. However, none of the previous studies identified human herpesvirus 8 in cutaneous lesions of primary systemic plasmacytosis (8). Hyperproduction of IL- 6 could be an important factor in the pathogenesis of cutaneous plasmacytosis because it induces B-cell proliferation and terminal differentiation.

Several treatments have been attempted, including topical and systemic corticosteroids, systemic antibiotics, and combined chemotherapies using agents such as melphalan, vincristine, cyclophosphamide, azathioprine, or even anti-CD20 antibody therapy, with poor clinical responses. On the other hand, topical tacrolimus and PUVA therapy have shown some improvement of skin lesions (9).

\section{Conclusions}

This exceedingly rare case highlights the unique features of a poorly understood plasmacytic disorder, supporting the concept that primary cutaneous plasmacytosis is a unique clinical entity.

\section{References}

1. Watanabe S, Ohara K, Kukita A, Mori S. Systemic plasmacytosis: a syndrome of peculiar multiple skin eruptions, generalized lymphadenopathy, and polyclonal hypergammaglobulinemia. Arch Dermatol. 1986;122:1314-20.

2. Han XD, Lee SSJ, Tan SH, Chong WS, Ng SK, Ooi MGM, et al. Cutaneous plasmacytosis: a clinicopathologic study of a series of cases and their treatment outcomes. Am J Dermatopathol. 2018;40:36-42.

3. Kitamura K, Tamura N, Hatano H, Toyama K, Mikata A, Watanabe S. A case of plasmacytosis with multiple peculiar eruptions. J Dermatol. 1980;7:341-9.

4. Uhara H, Saida T, Ikegawa S, Yamazaki Y, Mikoshiba H, Nijoh S, et al. Primary cutaneous plasmacytosis: report of three cases and review of the literature. Dermatol. 1994;189:251-5.

5. Leonard A, Meehan S, Ramsey D, Brown L, Sen F. Cutaneous and systemic plasmacytosis. J Am Acad Dermatol. 2007;56:38-40.

6. Nitta Y. Case of malignant lymphoma associated with primary systemic plasmacytosis with polyclonal hypergammaglobulinemia. Am J Dermatopathol. 1997; 19:289-93.

7. Honda R, Cerroni L, Tanikawa A, Ebihara T, Amagai M, Ishiko A. Cutaneous plasmacytosis: report of 6 cases with or without systemic involvement. J Am Acad Dermatol. 2013;68:978-85.

8. Miura H, Itami S, Yoshikawa K. Treatment of facial lesion of cutaneous plasmacytosis with tacrolimus ointment. J Am Acad Dermatol. 2003;39:1195-6. 